\title{
PROPUESTAS APLICADAS Y LÍNEAS DE INVESTIGACIÓN PARA LOS PROCESOS DE ADOPCIÓN DE MENORES
}

\author{
Eduardo Barca Enríquez \\ Departamento de Psicología. Universidad de A Coruña. \\ Orcid.org/0000-0003-2119-1849 \\ e.barcae@udc.es
}

Recepción Artículo: 22 octubre 2021

Admisión Evaluación: 22 octubre 2021

Informe Evaluador 1: 23 octubre 2021

Informe Evaluador 2: 24 octubre 2021

Aprobación Publicación: 24 octubre 2021

\section{RESUMEN}

La adopción de menores se ha convertido en España en un derecho del niño a tener una familia, destacando su propio interés para sus necesidades de desarrollo, tanto en su dimensión cognitiva, afectivo-social como personal y emocional. Así, el objetivo de este trabajo es explicar algunas actuaciones de intervención psicológica y psicosocial que se consideran relevantes en el ámbito de la adopción y acogimiento de menores. Por eso en este trabajo se exponen algunos de los resultados obtenidos a raíz de la investigación realizada por Barca en su tesis de doctorado (2015). En cuanto al método, se utilizan medidas de tendencia central y técnicas de análisis correlacionales. En los resultados obtenidos destacan la necesidad de equipos de trabajo e investigación sobre el impacto de las experiencias vitales dañinas en el niño y su remediación ya que son una garantía de que, desde el modelo de los Buenos Tratos, se llega al punto de partida obligado para lograr una fotografía psico-socioemocional del niño en la que se muestren las capacidades, las potencialidades y sus dificultades, así como las experiencias relativas a su recorrido vital que pueden estar en la base de muchas de las barreras evolutivas con las que se encuentra. Como conclusiones se considera que es imprescindible, en las actuaciones con estos menores, una formación especializada para los distintos profesionales que trabajan con el niño adoptado, tanto en el área educativa, como el área psicopedagógica y psicoterapéutica, para terminar haciendo unas propuestas para asegurar una "infancia segura".

Palabras clave: adopción internacional; deprivación temprana; intervención psicoemocional; institucionalización temprana; intervención psicopedagógica; psicoterapia

\section{ABSTRACT}

Applied proposals and lines of research for the processes of adoption of minors. The adoption of minors has become in Spain a child's right to have a family, highlighting their own interest for their 


\section{PROPUESTAS APLICADAS Y LÍNEAS DE INVESTIGACIÓN PARA LOS PROCESOS DE ADOPCIÓN DE MENORES}

development needs, both in their cognitive, affective-social and personal and emotional dimensions. Thus, the objective of this work is to explain some actions of psychological and psychosocial intervention that are considered relevant in the field of adoption and foster care of minors. For this reason, this work presents some of the results obtained as a result of the research carried out by Barca in his doctoral thesis (2015). Regarding the method, measures of central tendency and correlational analysis techniques are used. The results obtained highlight the need for work and research teams on the impact of harmful life experiences on the child and their remediation since they are a guarantee that, from the "Good Treatment" model, the obligatory starting point is reached to achieve a psycho-socio-emotional photograph of the child in which the capacities, potentialities and difficulties are shown, as well as the experiences related to their life path that may be at the base of many of the evolutionary barriers they encounter. As conclusions, it is considered that it is essential, in the actions with these minors, specialized training for the different professionals who work with the adopted child, both in the educational area, as well as the psychopedagogical and psychotherapeutic area, to end up making proposals to ensure a "Safe childhood".

Keywords: international adoption; early deprivation; psychoemotional intervention; early institutionalization; psychopedagogical intervention; psychotherapy

\section{INTRODUCCIÓN}

Resulta ya un deber y una obligación de todas las partes implicadas en el proceso de acogimiento, adoptivo y/o postadoptivo el hecho de poder aprovechar toda la investigación sobre adopción de menores y analizar los resultados obtenidos sobre las consecuencias que tienen muchas de las situaciones vividas por los niños y niñas en la etapa anterior al acogimiento y/o a la adopción y, así, poder incorporar todas las propuestas que desde muy diversos especialistas en el campo se proponen ya desde los últimos cinco años.

El contexto de adopciones se refiere a todo el proceso que viven los niños y las familias y que atañe al sistema de protección de menores de nuestro país y de los países de origen, donde se deben de tener formación continua y herramientas comunes y objetivas para valorar las situaciones de riesgo y actuar en consecuencia, conocer el nivel de daño sufrido por el niño y las posibles consecuencias en su desarrollo a corto y medio plazo, saber cómo valorar objetivamente a las familias de origen y a las acogedoras/adoptivas para poder ajustarse a las necesidades que el niño o niña pueda presentar, a las entidades mediadoras de todo el proceso de acogimiento y adoptivo y a los servicios de postadopción y acompañamiento en el acogimiento familiar para poder llevar a cabo un seguimiento profundo del nivel de desarrollo y evolución del mismo y de la familia como elemento nuclear en el sostén de las dinámicas complejas entre ambos si hubiera consecuencias conductuales graves.

Igualmente, la formación especializada en el ámbito de la traumaterapia infantil sistémica (Dantagnan, 2014) desde la administración para tener personal en el ámbito sanitario desde la psicología y la psiquiatría que pudieran Ilevar a nivel psicoterapéutico a aquellos que así lo requirieran, de una forma temprana y preventiva, ya que es habitual el peregrinaje por distintos profesionales y siempre desde el ámbito privado en la gran mayoría de los casos. También desde los centros de protección y reforma que atienden a tantos niños y los profesionales implicados, ya que ya en 2015 se superó en España la barrera de los 50.000 niños tutelados.

En el ámbito educativo, la formación de orientadores y personal de apoyo así como a todo el profesorado en la sensibilización de cómo estas vivencias tempranas implican consecuencias muchos años después de la adopción, y en algunos casos, con dramáticas consecuencias como puede ser aquellos con trastornos de apego reactivos que pueden ser precursores de patología mental posterior en la edad adulta. La educación obligatoria dura 10 años en España, y esos son muchos años para que puedan estar durante 3650 días sin la atención adecuada a las necesidades reales de estos chicos y chicas.

Sin duda desde la investigación, se deben de seguir investigando la evolución de los adultos de aquel "boom" de la adopción de hace ya más de 15 años y que ya muchos son mayores de edad y se conocen cómo se ajustan a las nuevas exigencias en los ámbitos familiares, laborales, sociales y comunitarios. 


\section{OBJETIVOS DE LA INVESTIGACIÓN}

En este trabajo se pretende hacer un compendio de las actuaciones que sería preciso tener presentes y llevar a cabo en el ámbito de la adopción y el acogimiento de menores a raíz de la investigación realizada por Barca (2015) en la que ha quedado patente la significativa influencia negativa que poseen las experiencias tempranas y todas las circunstancias previas a la adopción que se pueden encuadrar en el espectro del maltrato, el abuso y la negligencia.

Se busca, en definitiva, hacer una propuesta encaminada a la exposición y análisis de los diferentes procesos de intervención de tipo psicosocial y psicoeducativos dirigidos a los chicos adoptados y a sus familias.

\section{PARTICIPANTES}

La muestra estuvo integrada por 33 sujetos adoptados con un porcentaje de 51.5 de hombres y un 48.5 de mujeres y con edades comprendidas entre 5 y 17 años (Media 10.2; DT: 3.3). Cursan la mayor parte de los sujetos educación primaria y secundaria ( $65 \%$ y $35 \%$, respectivamente) con edades mayoritariamente entre los 5 y 7.6 años y los 7.7 y 11.6 años (ver tabla 1). Su procedencia es de África, Países del Este de Europa, de Latinoamérica y de Asia. Todos ellos Ilevaban conviviendo con sus familias adoptivas en el momento de realizar la investigación más de 5 años de duración, con residencia la mayoría en la provincia de A Coruña, en Galicia (ver tabla 2).

Tabla 1. Sexo y edades de los sujetos participantes

\begin{tabular}{lcclcc}
\hline Sexo & n & \% & Edades & n & $\%$ \\
\hline Mujeres & 16 & 48.5 & Entre 5 años y 7.6 años & 10 & 30.3 \\
Varones & 17 & 51.5 & Entre 7.7 años y11.6 años & 13 & 30.3 \\
Total & 33 & 100 & Entre 11.7 años y 14.6 años & 7 & 21.2 \\
& & Entre 14.7 años y 21 & 3 & 9.1 \\
& & Total & 33 & 100 \\
\hline
\end{tabular}

Tabla 2. Países de procedencia de los sujetos y Lugar de residencia actual

\begin{tabular}{llllll}
\hline Países procedencia & $\mathbf{n}$ & $\mathbf{\%}$ & Lugar de residencia & $\mathbf{n}$ & $\mathbf{\%}$ \\
\hline Etiopía & 11 & 33.3 & A Coruña ciudad & 13 & 39.4 \\
El Congo & 3 & 9.1 & A Coruña ciudad y limítrofes & 11 & 33.3 \\
China & 3 & 9.1 & Area Santiago Compostela & 4 & 12.1 \\
Nepal & 1 & 3 & Area Ferrol & 5 & 15.2 \\
Europa de Este & 9 & 27.2 & Total & 33 & 100 \\
Países Latinoamérica & 4 & 12.1 & & & \\
Nacional (España) & 2 & 6.1 & & & \\
Total & 33 & 100 & & & \\
& & & &
\end{tabular}

\section{MÉTODO}

\section{Instrumentos y análisis de datos}

En todo el proceso de obtención de datos se realizó de modo individualizado para cada sujeto que participa en la investigación y para cada familia. Antes de comenzar con la aplicación de las pruebas 
se ha tratado de obtener siempre los datos personales, de afiliación de padres y de los niños, así como de características preconsulta, durante la consulta y datos posteriores de seguimiento.

Los principales instrumentos de medida y obtención de datos fueron la Escala CIVIDA (Escala de análisis del itinerario vital) elaborada al efecto para la investigación. También se ha utilizado el test de Rutter (Escala de evaluación del contexto psicosocial) y, finalmente, la Batería de Evaluación Cognitiva (CAS) del Modelo PASS (Deaño, 2005).

Se han analizado los datos a través de medidas de tendencia central, con técnicas correlacionales y un análisis de varianza con el fin de buscar posibles diferencias estadísticamente significativas de variables de adopción y sus posibles incidencias en otras variables importantes de carácter escolar y conductual.

\section{RESULTADOS Y DISCUSIÓN}

Con los datos obtenidos ha sido posible configurar una serie de resultados que arrojan luz sobre el hecho de exponer y comprender los diferentes procesos de intervención con niños adoptados y sus familias desde diferentes áreas y de acuerdo con aportaciones de diversos autores con los que se harán varios análisis y discusión de resultados.

\section{EQUIPOS DE TRABAJO E INVESTIGACIÓN SOBRE EL IMPACTO DE LAS EXPERIENCIAS VITALES DAÑINAS EN EL NIÑO Y SU REMEDIACIÓN}

Para hacer formación adecuadamente sobre las consecuencias del trauma, es necesario llegar a conocer de manera exhaustiva las repercusiones que lo vivido tiene en el desarrollo del niño y el poder saber encontrar las herramientas más adecuadas para poder ayudarle a minimizar el impacto de lo vivido y favorecer esos recursos resilientes que todo niño lleva consigo (Giménez Alvira, 2013). Una evaluación comprehensiva de cada niño que incluya la evaluación de su estilo de apego, la presencia de experiencias traumáticas y su cualidad y el impacto que éstas tienen en el desarrollo (Barca, 2014; Barudy \& Dantagnan, 2005), es desde el Modelo de los "Buenos Tratos", un punto de partida obligado para poder tener la "radiografía psicosocioemocional" del niño en la que se muestren las capacidades, las potencialidades y sus dificultades, así como las experiencias relativas a su recorrido vital que pueden estar en la base de muchas de las barreras evolutivas con las que se encuentra. Un trabajo a destacar en esta línea es el desarrollado desde hace más de 20 años por el equipo del profesor Valentín Escudero desde el enfoque sistémico desde la Unidad de Investigación en Intervención y Cuidado Familiar en la Universidad de A Coruña.

Se hace necesario, por tanto, poder investigar cómo todo ese itinerario condiciona el desarrollo, en qué medida, con qué limitaciones, con qué posibilidades de recuperación, cómo estimular y construir nuevas vías de desarrollo de habilidades y destrezas y como aspecto fundamental, protocolos de intervención para padres, educadores y maestros, los agentes que mayor tiempo pasan con el niño, para poder atender a esas asincronías de la mejor manera posible, y no desde la intuición o el manual general, que suelen ser a los que se recurre inicialmente y que no suele tener los resultados deseados cuando estas dificultades son marcadas y se escapan de patrones conocidos.

\section{FORMACIÓN ESPECIALIZADA PARA LOS DISTINTOS PROFESIONALES QUE TRABAJAN CON EL NIÑO ADOPTADO}

\section{Área Educativa}

Siendo como era éste un grupo de niños adoptados con dificultades de diversa índole, personal, familiar y/o escolar, encontramos que éstas afectan en mayor o menor medida a su comportamiento y rendimiento. Aunque de los chicos que participaron en la investigación presentan un rendimiento académico bajo o muy bajo, solo un tercio, (13 de 33 suspendían una o más, que supone un $36 \%$ del grupo) es necesario tener en cuenta que, de los otros 20 chicos que señalan que aprueban todo, 0 bien justo, o bien sin mayores problemas, hay 8 padres ( 6 de 
Ios que aprueban todo) que refieren que su hijo recibe más nota de la que merece ya que parece que su rendimiento no se ajusta a las exigencias curriculares que están marcadas para su edad. Esto supondría casi un 20\% del grupo de participantes utilizado que sumado al anterior sería un $56 \%$ de la muestra con un rendimiento bajo o muy bajo. Esta es una realidad diaria donde padres y niños tienen una serie de retos educativos y personales que vienen marcados por algunas experiencias de su pasado, en el caso de los niños, y donde los padres necesitan un acompañamiento especializado por el enorme desafío que supone la crianza y educación de estos chicos.

Por ello, se considera que es necesario una mayor sensibilidad por parte de los equipos educativos a la hora de atender y entender las dificultades que presentan y que se muestran en el aula de muy diversa forma: bajo rendimiento, problemas en la adaptación y relación con los docentes, dificultades en la socialización con iguales y/o alteraciones en su comportamiento. Son varios los manuales específicos que nos ayudan a trabajar con una metodología eficaz en el aula con este tipo de chicos (Geddes, 2010), así como las orientaciones para maestros y profesores que ya han demostrado buenos resultados (Múgica, 2010).

Y una vez sensibilizados para poder entender que detrás de esas dificultades no hay incompetencias parentales ni problemas actitudinales sino unas crianzas de un elevado nivel de exigencia pero desde todos los actores, promover actividades de formación educativa especializada para saber cómo trabajar y atender las necesidades del "niño dañado" y que resumiría en tres grandes núcleos: 1) la identificación de sus dificultades desde una explicación comprehensiva y coherente con su recorrido vital, 2) el desarrollo de estrategias de relación y vinculación eficaces y cualitativamente distintas de las que conocemos y utilizamos actualmente para la mayoría de los niños, para promover con sus padres y educadores, y 3) la adaptación de contenidos curriculares y la creación de nuevas didácticas, estrategias de aprendizaje y metodologías ajustadas a sus necesidades reales, que igualmente, distan, en aquellos que presentan dificultades escolares, de las que se utilizan para el niño normal, estadísticamente hablando, y de las dificultades de aprendizaje que conocemos hasta el momento.

Todas estas propuestas estarían dirigidas para los confusos y desubicados padres (con razón y de manera muy legítima), que no acaban de saber cómo ayudar a sus hijos por las dificultades que entraña descodificarlas y darles respuesta. Y para los educadores que, en muchos casos, con más corazón que cabeza, intentan sacar a estos chicos adelante, aunque de manera infructuosa por desconocimiento, quizás, y la falta de herramientas en este campo tan específico y, en otros casos, no por ellos inexistentes, porque niegan las dificultades asociadas a historias vitales terribles a pesar de documentar e informar el impacto que estas tienen en el desarrollo cognitivo, emocional, social, personal y moral, no permitiendo ni asistiendo de la manera que necesitarían a estos y a sus familias.

El fin de estas medidas es permitir y favorecer el desarrollo de los verdaderos potenciales de esto chicos, que por sus experiencias tempranas traumáticas, en muchos casos quedan comprometidos las destrezas más relacionadas con lo académico y dejamos de lado sus otras inteligencias o habilidades (de excelentes condiciones físicas, artísticas en todas sus formas (música, pintura, baile, escritura...) y relacionales) que debieran ser rescatadas, ensalzadas y canalizadas hacia un desarrollo curricular que les permitiera poder enfocarlas hacia una enseñanzas educativas superiores 0 de inserción de empleo acorde con estas.

\section{Área Psicopedagógica y Psicoterapéutica}

También se hace necesaria una formación especializada a todo el grueso de profesionales externos a la escuela para que en cuanto se detecten los primeros problemas de adaptación escolar se tomen precozmente medidas de evaluación orientadas a poder establecer objetivamente los apoyos y las intervenciones necesarias, bien sean pedagógicas, psicopedagógicas, logopédicas y/o psicoterapéuticas para minimizar sus dificultades y maximizar sus logros. Siempre haciendo especial hincapié en la necesaria evaluación profunda y exhaustiva a la que ya hemos hechos referencia (Barca, 2014; Barudy \& Dantagnan, 2005; 2010), especialmente porque las cualidades diferenciales específicas de otros tipos de dificultades de aprendizaje y conducta, se apartan de un perfil concreto conocido pero que comparten características de muchas de ellas (Gonzalo, 2009). 
Esta necesidad puede ser uno de los motivos de que hayan surgido en diferentes ciudades españolas nuevos equipos de profesionales de carácter interdisciplinar con psicólogos, trabajadores sociales, psiquiatras, educadores, etc. que prestan servicios de ayuda y apoyo a estos niños y a sus familias adoptivas, como el IFIV de Barcelona dirigido por Jorge Barudy y Maryorie Dantagnan y la red Apega, con Gonzalo Marrodán y Rodríguez Borrajo como los pioneros en Bilbao y A Coruña, aunque actualmente ya se extiende por toda la geografía española y están presentes en el entramado técnico de algunas administraciones e instituciones que trabajan en la protección a la infancia y que siguen el paradigma del Buen Trato, la "Psicotraumatología Infantil Sistémica" y metodologías aplicadas coherentes (Barudy \& Dantagnan, 2011; Gonzalo, 2013) con resultados muy positivos y esperanzadores (Barudy \& Marquebreuq, 2009). Por otra parte, Jaime Ledesma y Anna Badía, dos profesionales ampliamente formados y especializados en el trabajo con niños y adultos adoptados, centran su intervención en el trabajo de mediación y búsqueda de orígenes para facilitar el complejo proceso de elaboración de la historia personal de estos chicos y chicas y establecer las condiciones más adecuadas para un posible reencuentro con su familia de origen.

\section{Área Psicosocial}

El trabajo que se realiza en este ámbito es cada vez más necesario donde el número de adoptados en el sistema de protección de menores va en aumento y siempre muy ligado a historias personales traumáticas, con situaciones de maltrato, abuso y/o negligencia severas y muy dañinas provocando problemas de salud mental que estarán detrás de la gran mayoría de estos casos.

El trabajo realizado por Jorge Barudy y Maryorie Dantagnan de reconocer el impacto que las experiencias traumáticas tienen en todas las áreas del desarrollo es de vital importancia para comprender de forma extensa la situación actual de cada niño y niña. Me gustaría destacar también el trabajo pionero en España hace ya 20 años en la intervención con trauma de Gabriella Bianco, a partir del trabajo psicoterapéutico que realizó con niños soldado en Alemania en los años 90. Y muy concretamente con su especialización en el ámbito de la Salud Mental Perinatal, dándole la importancia que tiene el trauma pero ya desde la concepción, desde el primer momento de la fecundación y durante todo el embarazo y en parto y postparto desde su centro Inanna en Palma de Mallorca, y en las diferentes universidades donde imparte formación en este campo.

Una discípula de esta excelente profesional, la psicóloga Carla Console y su compañera Xema Cabana, Trabajadora Social y Terapeuta Familiar, ha puesto en marcha en la Fundación Meniños desde 2017 un programa único en España de intervención y atención prenatal y perinatal con personas embarazadas en situación de riesgo psicosocial para prevenir ya desde los primeros momentos problemas de apego y evitar situaciones que puedan derivar en traumas para los menores ya desde que son un feto.

También en esta línea, el Programa Primera Alianza llevado a cabo por Carlos Pitillas y Ana Berástegui desde la Universidad Pontíficia de Comillas atiende a menores cuyo objetivo nuclear es fortalecer o reparar las relaciones afectivas tempranas entre niños en la edad preescolar y sus cuidadores primarios, en familias afectadas por diversas formas de trauma o en riesgo de exclusión social.

Quiero destacar también la fundamental labor realizada desde el asociacionismo para visibilizar y promover una mayor investigación y especialización de los profesionales que trabajan con niños y niñas adoptados y en acogimiento, como son las asociaciones de padres como Manaia en Galicia, las de adoptados como La Voz de Ios Adoptados, CORA, la coordinadora de asociaciones de adopción y acogimiento o las de profesionales como la cooperativa Agintzari, de las primeras en hablar de la necesidad de un trabajo terapéutico y psicosocial diferencial y específico con los niños y niñas víctimas de trauma y sus familias. También desde el ámbito universitario, destacar la labor del grupo AFIN de la Universidad Autónoma de Barcelona con figuras tan reconocidas como Diana Marré o Beatriz San Román, pioneras en la investigación con los menores acogidos y/o adoptados, desde un enfoque multidisciplinar aunando profesionales de muy distintos ámbitos. 


\section{PROPUESTAS PARA ASEGURAR UNA "INFANCIA SEGURA"}

Es una obligación de los gobiernos para el respeto de los derechos de la infancia, y para evitar mayores y costosos males, el promover las condiciones adecuadas en la institucionalización de niños mayores de tres años y promover el acogimiento familiar en los menores de tres años. En las situaciones donde esto no sea posible, será necesario invertir las energías y los recursos en mejorar estas condiciones, muy especialmente en los menores de tres años, por todo lo hasta ahora expuesto.

En este sentido, y debido a la vulnerabilidad física, fisiológica y psicológica de los niños en sus primeros años de vida, dependientes del vínculo con un adulto que provea el cuidado, afecto y estimulación necesarios para el adecuado desarrollo del menor (Rygaard, 2008), existe un movimiento muy importante en la promoción de buenas prácticas en las instituciones infantiles, como el proyecto "Fair Star" que tiene como objeto el mejorar la calidad de la atención a los niños menores de tres años. Señalan que son alrededor de 90.000 niños menores de tres años en toda Europa que están institucionalizados o en familias de acogida.

De ahí, la necesidad de dicho programa que tiene por objeto formar y supervisar tanto a los educadores como las condiciones y características de los centros y de su día a día, así como el acompañamiento a familias de acogida en la crianza con "base segura" de estos niños (Rygaard \& Husted, 2010). Aplaudimos e instamos a una mayor difusión y puesta en marcha de programas en esta dirección por el carácter preventivo y remediador que creemos que tiene sobre la salud infantil. Igualmente en la formación especializada de todos los profesionales del sistema de protección de menores que toman decisiones de vital relevancia para su futuro, así como las entidades implicadas en el acogimiento, la adopción y/o la postadopción, para asegurarles las mejores condiciones posibles de una forma lo más objetiva y consesuada de acuerdo a todo lo que ya conocemos sobre el "paradigma de los buenos tratos" de Jorge Barudy y Maryorie Dantagnan, así como los conocimientos acumulados sobre los efectos del trauma en los niños (Finkelhor, Ormrod, \& Turner, 2007) y el equipo de Noemí Pereda (Kanter \& Pereda, 2020) en la UAB sobre la Victimización Infantil y Adolescente y el inestimable legado que Boris Cyrulnik nos ha regalado en estos últimos veinte años desde el enfoque de la resiliencia.

Igualmente y siendo propuestas que atañen a los gobiernos y a los adultos que en ellos están, una medida para poder asegurar los procesos adoptivos tiene que ver con la ratificación del Convenio de la Haya, que asegura el cumplimiento de los derechos básicos de los menores y del compromiso del país para llevarlos a cabo. En 2011, los cinco principales países de origen de niños adoptados eran mayormente países no miembros: China (4.405), Etiopía (3.455), Rusia (3.325), Colombia (1.577) y Ucrania (1.070). Como señala Phillips (2013), estas cifras sólo reflejan las adopciones registradas, pero debemos tener en cuenta las numerosas adopciones ilegales que no son registradas y que se llevan a cabo especialmente por parte de personas que pertenecen a países que no adoptaron la Convención de La Haya. En 2011, los cinco principales países receptores de niños adoptados habían ratificado la Convención de la Haya: EUA (9.320), Italia (4.022), España (2.573), Francia (1.995) y Canadá (1.785).

Por lo tanto, como afirma Phillips (2013), es realmente importante que se ratifique la Convención de La Haya en todos los países posibles y la implementación de las disposiciones de la Convención es un paso vital para prevenir las adopciones ilegales e inseguras.

\section{REFERENCIAS BIBLIOGRÁFICAS}

Barca, E. (2014). Evaluación comprehensiva del niño adoptado: resignificando síntomas. En R. Ma․ Fernández García (Coord.) (2014). Neuropsicología del abandono y el maltrato infantil. Pp. 55-65. Barcelona: Hilo Rojo Ediciones.

Barca, E. (2015). Análisis del impacto y la alteración de la función cognitiva en niños adoptados y/o víctimas de maltrato, abuso y/o negligencia temprana desde el modelo PASS del procesamuento de la Información. Tesis doctoral, inédita. Universidad de Vigo.

Barudy, J. \& Dantagnan, M. (2005). Los buenos tratos a la infancia: Parentalidad, Apego y Resiliencia. Barcelona: Gedisa. 
Barudy, J. \& Dantagnan, M. (2010). Los desafíos invisibles de ser padre o madre: manual de evaluación de las competencias y la resiliencia parental. Barcelona: Gedisa.

Barudy, J. \& Marquebreuq, A.P. (2009). Hijas e hijos de madre resilientes. Barcelona: Gedisa.

Barudy, J., Dantagnan, M., Comas, E. \& Vergara, M. (2014). La inteligencia maternal. Manual para apoyar la crianza bien tratante y promover la resiliencia de madres y padres. Barcelona: Gedisa.

Deaño, M. (2005). D.N: CAS (Das. Naglieri: Sistema de Evaluación Cognitiva). Evaluación Española. Ourense: Gersam Ediciones.

Finkelhor, D., Ormrod, R. K., \& Turner, H. A. (2007). Polyvictimization and trauma in a national Iongitudinal cohort. Development and Psychopathology, 19, 149-166

Geddes, H. (2010). El apego en el aula: relación entre las primeras experiencias infantiles, el bienestar emocional y el rendimiento escolar. Barcelona: Graó.

Giménez Alvira, J.A. (2013). Indómito y Entrañable. Barcelona: Gedisa .ç

Gonzalo, J.L. (2009). Guía para el apoyo educativo de niños con trastornos del apego. Buenos Aires: Libros en Red.

Múgica, J. (2010). Hacia una escuela inclusiva: el reto de integrar niños y niñas socialmente desfavorecidos. Seminario para Berritzegune de Irún. Se puede consultar en línea en: http://g02.berritzeguneak.net/es/ (visto última vez el 29 de julio de 2015).

Kanter, B. \& Pereda, N. (2020). Victimización sexual en la infancia e intervención basada en la evidencia. Revista de psicoterapia, Vol. 31 (115), 197-212.

Phillips, C. (2013). Intercountry adoption: controversies and criticisms. International Association of Youth and Family Judges and Magistrates (AIMJF). Se puede consultar en www.charlottephillips.org/IAYFJM_201307.pdf

Rutter, M. (1998). The English and Romanian Adoptees Study Team. Developmental catch-up, and deficit, following adoption after severe global early privation. Journal of Child Psychology and Psychiatry, 39(4), 465-476.

Rygaard, N.P. (2008). El niño abandonado: guía para el tratamiento de los trastornos del apego. Barcelona: Gedisa.

Rygaard, N.P. \& Hustel, B. (2010). Manual para los usuarios del programa Fair Star: para directores de instituciones de atención infantil y profesores. Educational and Culture DG; Lifelong Learning Program. Comisión Europea. Se puede consultar en: http://www.train.fairstartedu.us/spain/handbook.pdf 